Pacific Journal of Mathematics

ON RIGHT ALTERNATIVE RINGS WITHOUT PROPER RIGHT 


\title{
ON RIGHT ALTERNATIVE RINGS WITHOUT PROPER RIGHT IDEALS
}

\section{ERWIN KLEINFELD}

\begin{abstract}
It is shown that a right alternative ring $R$ without proper right ideals, of characteristic not two, containing idempotents $e$ and $1, e \neq 1$, such that $e x=e(e x)$ for all $x \in R$ must be alternative and hence a Cayley vector matrix algebra of dimension 8 over its center.
\end{abstract}

In the classification of simple right alternative rings of characteristic not two it is still an open question whether there exist any which are not alternative, in contrast to characteristic two, where there do exist division rings which are not alternative [8]. A number of people have worked on this problem and were able to prove the alternative identity whenever they assumed an additional hypothesis such as finite dimensionality [1, 3], other identities [6, 7], or internal conditions on the ring $[4,5,9]$. It seems natural to try to tackle the case where there exists an idempotent $e \neq 1$ in $R$ such that $(e, e, R)=0$. If one could establish in this case that all simple $R$ of characteristic not 2 are alternative, then this would be a natural generalization of the theorem of Albert [2] for alternative rings, in which he showed that a simple alternative ring with idempotent $e \neq 1$ had to be either associative or a Cayley vector matrix algebra of dimension eight over its center.

In this paper we do not quite achieve this result, for we need to strengthen the hypothesis of simplicity to the assumption that the ring has no proper right ideals. On the other hand there is a good deal of information here that should prove useful in either romoving the hypothesis of $(e, e, R)=0$, or in constructing an example of a simple, right alternative ring of characteristic not two which is not alternative, if indeed such an example exists.

The main tool here is the fact that $(e, e, R)=0$ allows a Peirce decomposition into four "subspaces" $R_{i, j}, i, j=0,1$ as in the associative and alternative cases. The multiplication table for these subspaces differs in six places from the same table for alternative rings. By constructing appropriate right ideals we show in fact that the tables are the same. In the process we reduce the problem to the one studied by M. Humm-Kleinfeld [4], although by that time one can deduce from our work quite readily that indeed $R$ must be alternative.

2. Preliminary identities. In the course of the paper we require a number of identities which are true in arbitrary right alternative rings of characteristic not two: 
(1) $(a b, c, d)+(a, b,(c, d))=a(b, c, d)+(a, c, d) b$.

(2) $(x, a b, a)=(x, b, a) a$.

(3) $([a b] c) b=a([b c] b)$.

Proofs of these identities may be found on page 940 of [5].

(4) $(a b) c=a(b c)+a(c b)-(a c) b$,

also holds as this is the linearization of the right alternative identity.

3. Peirce decomposition. Henceforth in the paper, we assume that $R$ is a right alternative ring of characteristic not two, and that $R$ contains 1 and an idempotent of $e \neq 1$, such that $(e, e, R)=0$. If we define $R_{i j}=\{x \in R \mid e x=i x, x e=j x\}$ and $i, j=0,1$, then $R$ may be decomposed into a direct sum by $R=R_{11}+R_{10}+R_{01}+R_{00}$. HummKleinfeld has shown on page 166 [4] that the multiplication table of the $R_{i j}$ has the following containment properties:

\begin{tabular}{|c|c|c|c|c|}
\hline & $R_{11}$ & $R_{10}$ & $R_{01}$ & $R_{00}$ \\
\hline$R_{11}$ & $R_{11}+R_{01}$ & $R_{10}$ & $R_{10}$ & 0 \\
\hline$R_{10}$ & 0 & $R_{11}+R_{01}$ & $R_{11}$ & $R_{10}$ \\
\hline$R_{01}$ & $R_{01}$ & $R_{00}$ & $R_{00}+R_{10}$ & 0 \\
\hline$R_{00}$ & 0 & $R_{01}$ & $R_{01}$ & $R_{00}+R$ \\
\hline
\end{tabular}

Thus the first entry gives the information that $\left(R_{11}\right)^{2} \subset R_{11}+R_{01}$, etc. Besides, it is true that $x_{i i}^{2} \in R_{i i}$, and whenever $i \neq j$ that $x_{i j}^{2} \in R_{i i}$ as well as $x_{i j}^{3}=0$.

Throughout the paper whenever we need to refer to this result we shall use the phrase "it follows from the table that..."

We should bear in mind that in an alternative ring there are six places where stronger assertions can be made. These are: $\left(R_{11}\right)^{2} \subset R_{11}$, $R_{30}^{2} \subset R_{01}, R_{01}^{2} \subset R_{10},\left(R_{00}\right)^{2} \subset R_{00}, R_{11} R_{01}=0$, and $R_{00} R_{10}=0$.

\section{Main section.}

Lemma 1. In $R$ we have $\left(R_{11}^{2}\right)_{01} R_{10}=0$, and $\left(R_{11}^{2}\right)_{01} R_{01} \subset R_{10}$.

Proof. Let $x_{11}, y_{11} \in R_{11}, z_{10} \in R_{10}$. From the table it is obvious that $\left(x_{11}, z_{10}, y_{11}\right)=0$. Hence, using the right alternative identity,

$$
0=\left(x_{11}, y_{11}, z_{10}\right)=\left(x_{11} y_{11}\right) z_{10}-x_{11}\left(y_{11} z_{10}\right) \text {. }
$$

Let $x_{11} y_{11}=a_{11}+b_{01}$. Then, by substituting this in the previous equation, it follows that $a_{11} z_{10}+b_{01} z_{10}-x_{11}\left(y_{11} z_{10}\right)=0$, so that $b_{01} z_{10}=x_{11}\left(y_{11} z_{10}\right)-$ $a_{11} z_{10} \in R_{10} \cap R_{00}=0$, by use of the table. Hence, $b_{01} z_{10}=0$, thus proving the first part. Also let $z_{01} \in R_{01}$. Then 


$$
\left(x_{11}, y_{11}, z_{01}\right)=\left(x_{11} y_{11}\right) z_{01}-x_{11}\left(y_{11} z_{01}\right)=a_{11} z_{01}+b_{01} z_{01}-x_{11}\left(y_{11} z_{01}\right) .
$$

From the right alternative identity it follows that

$$
\left(x_{11}, y_{11}, z_{01}\right)=-\left(x_{11}, z_{01}, y_{11}\right)=-\left(x_{11} z_{01}\right) y_{11}+x_{11}\left(z_{01} y_{11}\right)=x_{11}\left(z_{01} y_{11}\right) \in R_{10},
$$

using the table. Hence solving the previous equation for $b_{01} z_{01}$, we see that $b_{01} z_{01}=\left(x_{11}, y_{11}, z_{01}\right)-a_{11} z_{01}+x_{11}\left(y_{11} z_{01}\right) \in R_{10}$, using the table. This completes the proof of the lemma.

Definition. Let $T_{01}=\left\{x_{01} \in R_{01} \mid x_{01} R_{10}=0\right.$, and $\left.x_{01} R_{01} \subset R_{10}\right\}$ and form $T=T_{01}+T_{01} R_{01}+\cdots+\left(\cdots\left(T_{01} R_{01}\right) R_{01} \cdots\right) R_{01}+\cdots+$ where each term except the first is obtained from the preceding by right multiplication by $R_{01}$.

Lemma 2. $T$ is a right ideal of $R$ such that $T \subset R_{01}+R_{10}+R_{11}$.

Proof. For arbitrary $t_{01} \in T_{01}, x_{11} \in R_{11}, y_{10} \in R_{10}$ and $z_{01} \in R_{01}$ we have $\left(t_{01} x_{11}\right) y_{10}=\left(t_{01}, x_{11}, y_{10}\right)=-\left(t_{01}, y_{10}, x_{11}\right)=-\left(t_{01} y_{10}\right) x_{11}+t_{01}\left(y_{10} x_{11}\right)=0$,

using the right alternative identity and the definition of $T_{01}$, as well as the table. Also,

$$
\begin{aligned}
\left(t_{01} x_{11}\right) z_{01} & =\left(t_{01}, x_{11}, z_{01}\right)+t_{01}\left(x_{11} z_{01}\right)=\left(t_{01}, x_{11}, z_{01}\right) \\
& =-\left(t_{01}, z_{01}, x_{11}\right)=-\left(t_{01} z_{01}\right) x_{11}+t_{01}\left(z_{01} x_{11}\right) \\
& =a_{10} x_{11}+t_{01}\left(z_{01} x_{11}\right)=t_{01}\left(z_{01} x_{11}\right) \in R_{10}
\end{aligned}
$$

using the same reasons as before. But then $t_{01} x_{11} \in T_{01}$ and thus $T_{01} R_{11} \subset$ $T_{01}$. Also, from the definition of $T_{01}$ it follows almost immediately that $T_{01} R_{10}=0$, and $T_{01} R_{01} \subset R_{10}$, while the table implies that $T_{01} R_{00}=0$. Let $P(n)$ be the $n+1^{s t}$ term in the sum that defines $T$, and let $U(n)=$ $T_{01}+T_{01} R_{01}+\cdots+P(n)$, be the sum of the first $n+1$ terms in the definition of $T$. We shall prove by induction that $P(n) R_{11} \subset U(n)$, $P(n) R_{10} \subset U(n)$ and $P(n) R_{00} \subset U(n)$. We have already seen this is true for $n=0$. Assume it is true for $n$ and then we shall prove it true for $n+1$. We abbreviate $P(n)$ by simply $P$. Then using (4), and the table, $\left(P R_{01}\right) R_{11} \subset P\left(R_{01} R_{11}\right)+P\left(R_{11} R_{01}\right)+\left(P R_{11}\right) R_{01} \subset P R_{01}+P R_{10}+$ $\left(P R_{11}\right) R_{01} \subset P(n+1)+U(n)+U(n) R_{01} \subset U(n+1)$. Also similarly,

$$
\begin{aligned}
\left(P R_{01}\right) R_{10} \subset P\left(R_{01} R_{10}\right) & +P\left(R_{10} R_{01}\right)+\left(P R_{10}\right) R_{01} \subset P R_{00}+P R_{11} \\
& +\left(P R_{10}\right) R_{01} \subset U(n)+U(n) R_{01} \subset U(n+1),
\end{aligned}
$$

and

$$
\begin{aligned}
\left(P R_{01}\right) R_{00} \subset P\left(R_{01} R_{00}\right) & +P\left(R_{00} R_{01}\right)+\left(P R_{00}\right) R_{01} \subset P R_{01} \\
& +\left(P R_{00}\right) R_{01} \subset P(n+1)+U(n) R_{01} \subset U(n+1) .
\end{aligned}
$$


Consequently, $P(n+1) A \subset U(n+1)$ for $A=R_{11}, R_{10}$, and $R_{00}$. This completes the induction. But then, $T A \subset T$. Of course, also $T R_{01} \subset T$. But then, $T R \subset T$ and, hence, $T$ is a right ideal of $R$. Also, $P(1)=$ $T_{01} R_{01} \subset R_{10}$ by definition of $T_{01}$. Hence $P(2) \subset R_{10} R_{01} \subset R_{11}$, and so $P(3) \subset R_{11} R_{01} \subset R_{10}$. Thence $P(2 n+1) \subset R_{10}$ and $P(2 n) \subset R_{11}$, so that $T \subset T_{01}+R_{10}+R_{11} \subset R_{01}+R_{10}+R_{11}$. This completes the proof of the lemma.

We note that there is complete symmetry if the idempotent $e$ is replaced by the idempotent $1-e$. In terms of the Peirce decomposition this has the effect of simply permuting subscripts. We shall frequently use this play in order to obtain new results from theorems already proved, and justify it by stating that "we may reverse subscripts...." Thus we may assert:

CoRollaRy 1. If $R$ has no proper right ideals then $R_{11}^{2} \subset R_{11}$.

CoRollaRy 2. If $R$ has no proper right ideals then $R_{00}^{2} \subset R_{00}$.

Proof. The right ideal $T$ of Lemma 2 cannot be $R$ since $1-e \in R_{00}$ would then have to be zero, contrary to assumption. But then $T=0$, hence $T_{01}=0$. But Lemma 1 implies that $\left(R_{11}^{2}\right)_{01} \in T_{01}$, so that $\left(R_{11}^{2}\right)_{01}=0$, hence $R_{11}^{2} \subset R_{11}$. But then we may reverse subscripts and obtain the second corollary as well.

In the remainder of the paper we shall assume tacitly that, in addition, $R$ has no proper right ideals, so that we may freely use the results of the last two corollaries.

LEMMA 3. $R_{11}$ is associative.

Proof. Let $A=\sum\left(R_{11}, R_{11}, R_{11}\right)+R_{11}\left(R_{11}, R_{11}, R_{11}\right)$. Since $R_{10} R_{11}=0$ follows from the table, while $R_{11}^{2} \subset R_{11}$ because of Corollary 1 , we can easily verify that $\left(R_{10}, R_{11}, R_{11}\right)=0$. Select $w_{11}, y_{11}, z_{11}, \in R_{11}$ and $x_{10} \in R_{10}$. Then substitute $a=w_{11}, b=x_{10}, c=y_{11}, d=z_{11}$ in (1), obtaining

$$
\left(w_{11} x_{10}, y_{11}, z_{11}\right)+\left(w_{11}, x_{10},\left(y_{11}, z_{11}\right)\right)=w_{11}\left(x_{10}, y_{11}, z_{11}\right)+\left(w_{11}, y_{11}, z_{11}\right) x_{10} \text {. }
$$

However, by inspection $\left(R_{11}, R_{10}, R_{11}\right)=0$, as a consequence of the table, so that only one term survives in the preceding equation. Thus $\left(R_{11}, R_{11}, R_{11}\right) R_{10}=0$. We have already observed that $\left(R_{11}, R_{10}, R_{11}\right)=0$. If we apply the right alternative identity in this situation then it follows that $\left(R_{11}, R_{11}, R_{10}\right)=0$, and hence $\left(R_{11},\left(R_{11}, R_{11}, R_{11}\right), R_{10}\right)=0$. Expanding the last associator, thus $R_{11}\left(R_{11}, R_{11}, R_{11}\right) \cdot R_{10}=0$. But then, 
$A R_{10}=0$. Since $A \subset R_{11}$, it follows from the table that $A R_{00}=0$. Besides, it is well known that even if $R_{11}$ where an arbitrary ring, not necessarily right alternative, that $A$ is always a two-sided ideal of $R_{11}$, so that $A R_{11} \subset A$. Let us form

$$
B=A+A R_{01}+\left(A R_{01}\right) R_{01}+\cdots+\left(\cdots\left(A R_{01}\right) R_{01} \cdots\right)+\cdots
$$

where the $n^{\text {th }}$ term is obtained from the preceding by right multiplication by $R_{01}$, except for $n=1$. As in the proof of Lemma 2 the reader may easily check that $B$ is a right ideal of $R$ using induction. But the odd terms in the equation defining $B$ are contained in $R_{11}$, while the even terms are contained in $R_{10}$, using the table. Hence, $B \subset R_{10}+R_{11}$. Since $B=R$ implies $1-e=0$, we must have $B=0$, hence $A=0$. Thus $R_{11}$ is associative, completing the proof of the lemma.

COROLlary. $R_{00}$ is associative.

Proof. We may reverse subscripts in the lemma.

LemMa 4 . ( i ) $R_{11}+R_{01}=R_{10} R_{01}+R_{10}^{2}+R_{00} R_{10}$.

(ii) $R_{00}+R_{10}=R_{01} R_{10}+R_{01}^{2}+R_{11} R_{01}$.

(iii) $R_{00}=R_{01} R_{10}$.

(iv) $R_{11}=R_{10} R_{01}$.

Proof. Define inductively $R_{10}^{n}=R_{10}^{n-1} R_{10}$ and form $A=R_{10} R_{01}+$ $R_{10}+\cdots+R_{10}^{n}+\cdots$. First we aim to show that $A$ must be a right ideal of $R$. By repeated use of (4) and table we see that

$$
\begin{aligned}
& \left(R_{10} R_{01}\right) R_{11} \subset R_{10}\left(R_{01} R_{11}+R_{11} R_{01}\right)+\left(R_{10} R_{11}\right) R_{01} \subset R_{10} R_{01}+R_{10}^{2}, \\
& \left(R_{10} R_{01}\right) R_{10} \subset R_{11} R_{10} \subset R_{10},\left(R_{10} R_{01}\right) R_{01} \subset R_{11} R_{01} \subset R_{10}, \\
& \left(R_{10} R_{01}\right) R_{00} \subset R_{11} R_{00}=0,
\end{aligned}
$$

thus showing that $\left(R_{10} R_{01}\right) R \subset R_{10} R_{01}+R_{10}+R_{10}^{2} \subset A$. Also $R_{10} R_{11}=0$, $R_{10} R_{10}=R_{10}^{2}, R_{10} R_{01} \subset A, R_{10} R_{00} \subset R_{10}$, using the table. But use of (4) and the table shows that

$$
\begin{gathered}
\left(R_{10}^{2}\right) R_{11} \subset R_{10}\left(R_{10} R_{11}+R_{11} R_{10}\right)+\left(R_{10} R_{11}\right) R_{10} \subset R_{10}^{2}, R_{10}^{2} R_{10}=R_{10}^{3}, \\
\left(R_{10}^{2}\right) R_{01} \subset R_{10}\left(R_{10} R_{01}+R_{01} R_{10}\right)+\left(R_{10} R_{01}\right) R_{10} \subset R_{10} R_{11} \\
+R_{10} R_{00}+R_{11} R_{10} \subset R_{10},
\end{gathered}
$$

while $\left(R_{10}^{2}\right) R_{00} \subset\left(R_{11}+R_{01}\right) R_{00}=0$. Now define

$$
Q(n)=R_{10} R_{01}+R_{10}+\cdots+R_{10}^{n} .
$$

The above calculations show that $Q(2) B \subset Q(2)$, for $B=R_{11}, R_{01}$ and 
$R_{00}$. Assume inductively that $R_{10}^{n} B \subset Q(n)$ and we proceed to prove this inclusion for $n+1$ in place of $n$. Besides the induction hypothesis, our main tools are (4) and the table. $\left(R_{10}^{n} R_{10}\right) R_{11} \subset R_{10}^{n}\left(R_{10} R_{11}+R_{11} R_{10}\right)+$ $\left(R_{10}^{n} R_{11}\right) R_{10} \subset R_{10}^{n} R_{10}+\left(R_{10}^{n} R_{11}\right) R_{10} \subset R_{10}^{n+1}+Q(n) R_{10} \subset Q(n+1)$. Similarly,

$$
\begin{aligned}
\left(R_{10}^{n} R_{10}\right) R_{01} \subset R_{10}^{n}\left(R_{10} R_{01}\right. & \left.+R_{01} R_{10}\right)+\left(R_{10}^{n} R_{01}\right) R_{10} \subset R_{10}^{n} R_{11}+R_{10}^{n} R_{00} \\
& +Q(n) R_{10} \subset Q(n)+Q(n+1) \subset Q(n+1) .
\end{aligned}
$$

Finally, $\left(R_{10}^{n} R_{10}\right) R_{00} \subset R_{10}^{n}\left(R_{10} R_{00}+R_{00} R_{10}\right)+\left(R_{10}^{n} R_{00}\right) R_{10} \subset R_{10}^{n} R_{10}+R_{10}^{n} R_{01}+$ $Q(n) R_{10} \subset Q(n+1)$. This completes the induction. Armed with this information we are now ready to prove that $A$ is a right ideal of $R$. Since $Q(2) B \subset Q(2) \subset A$ and $R_{10}^{n} B \subset Q(n) \subset A$, we see that $A B \subset A$. Since obviously $R_{10}^{n} R_{10}=R_{10}^{n+1}$, it follows also that $A R_{10} \subset A$. But then $A R \subset A$, and thus $A$ is a right ideal of $R$. Let us consider first the case $A=0$. In that case, $R_{10}=0$. Form $B=R_{00}+R_{01}$. Using the table and Corollary 2 of Lemma 2 , we may varify that $B$ is a right ideal. Since $e \notin B$, we must then have $B=0$. But then $R=R_{11}$, so that $e=1$, contrary to assumption. Hence the case $A=0$ cannot arise. The only open possibility is that $A=R$. Now from the table we see that $R_{10}^{2} \subset R_{11}+R_{01}$, while $R_{10}^{3} \subset\left(R_{11}+R_{01}\right) R_{10} \subset R_{10}+R_{00}$, and $\left(R_{10}+R_{00}\right) R_{10} \subset$ $R_{11}+R_{01}$. Consequently, $R_{10}^{2 n} \subset R_{11}+R_{01}$, and $R_{10}^{2 n+1} \subset R_{10}+R_{00}$, for all positive integers $n$. Since the Peirce decomposition is direct and $A=R$, it must be that $R_{11}+R_{01}=\sum R_{10}^{2 n}+R_{10} R_{01}$, and $R_{10}+R_{00}=\sum R_{10}^{2 n-1}$. But note that by definition $R_{10}^{2 n+2}=\left(R_{10}^{2 n} R_{10}\right) R_{10} \subset\left(\left[R_{11}+R_{01}\right] R_{10}\right) R_{10} \subset R_{10}^{2}+$ $R_{00} R_{10}$ and so $\sum R_{10}^{2 n} \subset R_{10}^{2}+R_{00} R_{10}$. But then from two equations back it follows that $R_{11}+R_{01} \subset R_{10}^{2}+R_{00} R_{10}+R_{10} R_{01}$. On the other hand it is a consequence of the table that $R_{10}^{2}+R_{00} R_{10}+R_{10} R_{01} \subset R_{11}+R_{01}$, so that $R_{11}+R_{01}=R_{10}^{2}+R_{00} R_{10}+R_{10} R_{01}$. This establishes part (i). To obtain (ii) from (i), simply reverse subscripts. By definition

$$
\begin{aligned}
& R_{10}^{2 n+1} \subset\left(R_{10}^{2 n-1} R_{10}\right) R_{10} \subset\left(\left[R_{10}+R_{00}\right] R_{10}\right) R_{10} \subset \\
& R_{10}^{3}+R_{01} R_{10} \subset\left(R_{11}+R_{01}\right) R_{10}+R_{01} R_{10} \subset R_{10}+R_{01} R_{10} .
\end{aligned}
$$

But then $R_{10}+R_{00}=\sum R_{10}^{2 n-1} \subset R_{10}+R_{01} R_{10} \subset R_{10}+R_{00}$. But then $R_{10}+$ $R_{01} R_{10}=R_{10}+R_{00}$. Using the directness of the Peirce decomposition we obtain that $R_{01} R_{10}=R_{00}$. This establishes part (iii). Part (iv) follows from part (iii) by reversing subscripts. This completes the proof of the lemma.

LemmA 5. For all $a_{01} \in R_{01}$ and $x_{11}, y_{11} \in R_{11},\left(a_{01} x_{11}\right) y_{11}=a_{01}\left(y_{11} x_{11}\right)$.

Proof. It follows from Lemma 4 -(i) that $a_{01} \in R_{10} R_{01}+R_{10}^{2}+R_{00} R_{10}$. Using (4) and the table we see that for

$$
b_{10}, c_{10} \in R_{10},\left(b_{10} c_{10}\right) x_{11}=b_{10}\left(c_{10} x_{11}+x_{11} c_{10}\right)-\left(b_{10} x_{11}\right) c_{10}=b_{10}\left(x_{11} c_{10}\right) .
$$


By repeated use of this last equation, then $\left(\left[b_{10} c_{10}\right] x_{11}\right) y_{11}=\left(b_{10}\left[x_{11} c_{10}\right]\right) y_{11}=$ $b_{10}\left(y_{11}\left[x_{11} c_{10}\right]\right)$. As previously observed, $\left(y_{11}, x_{11}, c_{10}\right)=-\left(y_{11}, c_{10}, x_{11}\right)=0$. Thus, $b_{10}\left(y_{11}\left[x_{11} c_{10}\right]\right)=b_{10}\left(\left[y_{11} x_{11}\right] c_{10}\right)=\left(b_{10} c_{10}\right)\left(y_{11} x_{11}\right)$ using the table, Lemma 2-Corollary 1, and the previous observation we made use of just before. Combining two previous equations, we see that $\left(\left[b_{10} c_{10}\right] x_{11}\right) y_{11}=\left(b_{10} c_{10}\right)$ $\left(y_{11} x_{11}\right)$. Thus $b_{10} c_{10}$ has the desired property. Let $b_{00} \in R_{00}$. Then (4) and the table imply that $\left(b_{00} c_{10}\right) x_{11}=b_{00}\left(c_{10} x_{11}+x_{11} c_{10}\right)-\left(b_{00} x_{11}\right) c_{10}=b_{00}\left(x_{11} c_{10}\right)$. Using the table, Lemma 2-Corollary 1 , and the previous equation repeatedly, it follows that $\left(\left[b_{00} c_{10}\right] x_{11}\right) y_{11}=\left(b_{00}\left[x_{11} c_{10}\right]\right) y_{11}=b_{00}\left(y_{11}\left[x_{11} c_{10}\right]\right)$. As already noted, $\left(y_{11}, x_{11}, c_{10}\right)=0$, so that $b_{00}\left(y_{11}\left[x_{11} c_{10}\right]\right)=b_{00}\left(\left[y_{11} x_{11}\right] c_{10}\right)=$ $\left(b_{00} c_{10}\right)\left(y_{11} x_{11}\right)$. Thus $b_{00} c_{10}$ has the desired property. Finally, if $z_{11} \in R_{11}$, then $\left(z_{11} x_{11}\right) y_{11}-z_{11}\left(y_{11} x_{11}\right) \in R_{11}$ because of Lemma 2-Corollary 1 . Hence, $\left(a_{01} x_{11}\right) y_{11}-a_{01}\left(y_{11} x_{11}\right) \in R_{11}$, But from the table it follows that $\left(a_{01} x_{11}\right) y_{11}$ $a_{01}\left(y_{11} x_{11}\right) \in R_{01}$. Since $R_{01} \cap R_{11}=0$, it must be that $\left(a_{01} x_{11}\right) y_{11}-a_{01}\left(y_{11} x_{11}\right)=$ 0 . This completes the proof of the lemma.

LeMma $6 . \quad R_{11}$ and $R_{00}$ are commutative.

Proof. Let $a_{01} \in R_{01}, b_{10} \in R_{10}$ and $x_{11}, y_{11} \in R_{11}$. As a result of (1), $\left(b_{10} a_{01}, x_{11}, y_{11}\right)+\left(b_{10}, a_{01},\left(x_{11}, y_{11}\right)\right)=b_{10}\left(a_{01}, x_{11}, y_{11}\right)+\left(b_{10}, x_{11}, y_{11}\right) a_{01}$. Use of the table reveals that $\left(b_{10}, x_{11}, y_{11}\right)=0$, since $R_{11}$ is a subring. Moreover, Lemma 3 and the table imply that $\left(b_{10} a_{01}, x_{11}, y_{11}\right)=0$. Thus only two terms survive in the first equation and we see that $\left(b_{10}, a_{01},\left(x_{11}, y_{11}\right)\right)=$ $b_{10}\left(a_{01}, x_{11}, y_{11}\right)$. Moreover,

$$
\left(b_{10}, a_{01},\left(x_{11}, y_{11}\right)\right)=\left(b_{10} a_{01}\right)\left(x_{11} y_{11}-y_{11} x_{11}\right)-b_{10}\left[a_{01}\left(x_{11} y_{11}-y_{11} x_{11}\right)\right],
$$

expanding the associator. But

$$
\begin{array}{r}
-b_{10}\left[a_{01}\left(x_{11} y_{11}-y_{11} x_{11}\right)\right]=-b_{10}\left[\left(a_{01} y_{11}\right) x_{11}-a_{01}\left(y_{11} x_{11}\right)\right] \\
=-b_{10}\left(a_{01}, y_{11}, x_{11}\right)=b_{10}\left(a_{01}, x_{11}, y_{11}\right),
\end{array}
$$

using Lemma 5 and the right alternative identity. Now if we compare the last three equations we conclude that $\left(b_{10} a_{01}\right)\left(x_{11} y_{11}-y_{11} x_{11}\right)=0$. At this point Lemma 4-(iv) may be utilized to conclude that for every $z_{11} \in R_{11}, z_{11}\left(x_{11} y_{11}-y_{11} x_{11}\right)=0$. In particular we may choose $z_{11}=e$. Then because of Lemma 2-Corollary $1, x_{11} y_{11}-y_{11} x_{11}=0$. Thus $R_{11}$ is seen to be commutative. By reversing subscripts, it follows that $R_{00}$ is also commutative. This completes the proof of the lemma.

\section{Lemma 7. $\left(R_{01}, R_{11}, R_{11}\right)=0=\left(R_{10}, R_{00}, R_{00}\right)$.}

Proof. Let $a_{01} \in R_{01}$ and $x_{11}, y_{11} \in R_{11}$. Then because of Lemmas 5 and 6, $\left(a_{01} x_{11}\right) y_{11}=a_{01}\left(y_{11} x_{11}\right)=a_{01}\left(x_{11} y_{11}\right)$, thus establishing $\left(a_{01}, x_{11}, y_{11}\right)=0$. Hence, $\left(R_{01}, R_{11}, R_{11}\right)=0$. By reversing subscripts, $\left(R_{10}, R_{00}, R_{00}\right)=0$ follows. This completes the proof of the lemma. 
Definition. We define $Q_{00}=$ nilpotent elements of $R_{00}$, and $Q_{11}=$ nilpotent elements of $R_{11}$.

We note, since $R_{11}, R_{00}$ are associative, commutative, subrings of $R$, that $Q_{11}$ is an ideal of $R_{11}$ and $Q_{00}$ an ideal of $R_{00}$.

LEMMA 8. If $a_{11} \in R_{11}, b_{01} \in R_{01}, c_{10} \in R_{10}$, then

$$
d_{11}=\left(a_{11} b_{01}\right) c_{10}=\left(a_{11}, b_{01}, c_{10}\right)=-\left(a_{11}, c_{10}, b_{01}\right)
$$

satisfies $d_{11}^{2}=0$, so that $d_{11} \in Q_{11}$. Similarly, if

$$
d_{00}=\left(a_{00} b_{10}\right) c_{01}=\left(a_{00}, b_{10}, c_{01}\right)=-\left(a_{00}, c_{01}, b_{10}\right),
$$

then $d_{00}^{2}=0$ and $d_{00} \in Q_{00}$. Thus $\left(R_{11} R_{01}\right) R_{10} \subset Q_{11}$ and $\left(R_{00} R_{10}\right) R_{01} \subset Q_{00}$.

Proof. As a result of (4), $\left(a_{11} b_{01}\right) c_{10}=a_{11}\left(b_{01} c_{10}+c_{10} b_{01}\right)-\left(a_{11} c_{10}\right) b_{01}$. But $a_{11}\left(b_{01} c_{10}\right) \in R_{11} R_{00}=0$, from the table, while $a_{11}\left(c_{10} b_{01}\right) \in R_{11}^{2} \subset R_{11}$, - $\left(a_{11} c_{10}\right) b_{01} \in R_{10} R_{01} \subset R_{11}$, using the table and Lemma 2-Corollary. Hence $\left(a_{11} b_{01}\right) c_{10} \in R_{11}$ and so $\left(R_{11} R_{01}\right) R_{10} \subset R_{11}$. Let $f_{10}=a_{11} b_{01}$ and $d_{11}=\left(a_{11} b_{01}\right) c_{10}$. Then $f_{10} c_{10}=d_{11}$, while $d_{11}^{2}=\left(f_{10} c_{10}\right) d_{11}=f_{10}\left(c_{10} d_{11}+d_{11} c_{10}\right)-\left(f_{10} d_{11}\right) c_{10}$, using (4). Since $R_{10} R_{11}=0$, follows from the table, two terms vanish in the last equation, so that $d_{11}^{2}=f_{10}\left(d_{11} c_{10}\right)$. But $f_{10}\left(d_{11} c_{10}\right)=f_{10}\left(\left[f_{10} c_{10}\right] c_{10}\right)=$ $f_{10}\left(f_{10}\left[c_{10}^{2}\right]\right)$, because of the right alternative identity. Since

$$
f_{10}\left(c_{10}^{2}\right) \in R_{10} R_{11}=0,
$$

as a result of the table, it follows that $d_{11}^{2}=0$, and so $d_{11} \in Q_{11}$. By interchanging subscripts we obtain the second part. This completes the proof of the lemma.

LEMMA 9. Let

$$
\begin{aligned}
Q= & Q_{11}+Q_{11} R_{10}+R_{01} Q_{11}+R_{01} Q_{11} R_{10}+Q_{00}+Q_{00} R_{01}+R_{10} Q_{00} \\
& +R_{10} Q_{00} R_{01}+R_{11} R_{01}+\left(R_{11} R_{01}\right) R_{01}+R_{00} R_{10}+\left(R_{00} R_{10}\right) R_{10}
\end{aligned}
$$

Then $Q$ is a right ideal of $R$.

Proof. Most of the calculations involved are routine, and (4) is an important tool. Unless the reasoning is complicated, we shall state the appropriate inclusions without comment. $\left(R_{11} R_{01}\right) R_{11} \subset R_{10} R_{11}=0$. $\left(R_{11} R_{01}\right) R_{10} \subset Q_{11}$, because of Lemma 8. $\quad\left(R_{11} R_{01}\right) R_{01} \subset Q . \quad\left(R_{11} R_{01}\right) R_{00} \subset$ $R_{11}\left(R_{01} R_{00}+R_{00} R_{01}\right)+\left(R_{11} R_{00}\right) R_{01} \subset R_{11}\left(R_{00} R_{01}\right) \subset R_{11} R_{01}$, using (4) and the table.

$$
\begin{aligned}
& \left(\left[R_{11} R_{01}\right] R_{01}\right) R_{11} \subset\left(R_{11} R_{01}\right)\left(R_{01} R_{11}+R_{11} R_{01}\right) \\
& \quad+\left(\left[R_{11} R_{01}\right] R_{11}\right) R_{01} \subset\left(R_{11} R_{01}\right) R_{01}+\left(R_{11} R_{01}\right) R_{10}+\left(\left[R_{11} R_{01}\right] R_{11}\right) R_{01}
\end{aligned}
$$


using (4). Now $\left(R_{11} R_{01}\right) R_{10} \subset Q_{11}$ because of Lemma 8, while we observed earlier in the proof that $\left(R_{11} R_{01}\right) R_{11}=0$. Consequently,

$$
\begin{aligned}
& \left(\left[R_{11} R_{01}\right] R_{01}\right) R_{11} \subset\left(R_{11} R_{01}\right) R_{01}+Q_{11} . \\
& \left(\left[R_{11} R_{01}\right] R_{01}\right) R_{10} \subset\left(R_{11} R_{01}\right)\left(R_{01} R_{10}+R_{10} R_{01}\right) \\
& \quad+\left(\left[R_{11} R_{01}\right] R_{10}\right) R_{01} \subset\left(R_{11} R_{01}\right) R_{00}+\left(R_{11} R_{01}\right) R_{11}+\left(\left[R_{11} R_{01}\right] R_{10}\right) R_{01},
\end{aligned}
$$

using (4). But we have already observed that $\left(R_{11} R_{01}\right) R_{00} \subset R_{11} R_{01}$, and $\left(R_{11} R_{01}\right) R_{11}=0$, while Lemma 8 implies $\left(R_{11} R_{01}\right) R_{10} \subset R_{11}$, so that

$$
\left(\left[R_{11} R_{01}\right] R_{10}\right) R_{01} \subset R_{11} R_{01} \text {. }
$$

Combining these observations it follows that $\left(\left[R_{11} R_{01}\right] R_{01}\right) R_{10} \subset R_{11} R_{01}$. $\left(\left[R_{11} R_{01}\right] R_{01}\right) R_{01} \subset\left(R_{10} R_{01}\right) R_{01} \subset R_{11} R_{01}$.

$$
\left(\left[R_{11} R_{01}\right] R_{01}\right) R_{00} \subset\left(R_{10} R_{01}\right) R_{00} \subset R_{11} R_{00}=0 .
$$

As we have already observed, $Q_{11}$ is an ideal of $R_{11}$, and so $Q_{11} R_{11} \subset Q_{11}$. $Q_{11} R_{10} \subset Q . \quad Q_{11} R_{01} \subset R_{11} R_{01} . \quad Q_{11} R_{00}=0 . \quad\left(Q_{11} R_{10}\right) R_{11} \subset R_{10} R_{11}=0$. In order to obtain the desired inclusion for $\left(Q_{11} R_{10}\right) R_{10}$, we observe first that $a_{10}^{3}=0$ follows from the table, hence $a_{10}^{4}=0$, so that $a_{10}^{2} \in Q_{11}$. By linearization, then $a_{10} b_{10}+b_{10} a_{10} \in Q_{11}$. If $q_{11} \in Q_{11}$, then $q_{11} a_{10} \in R_{10}$, so that $\left(q_{11} a_{10}\right) b_{10}+b_{10}\left(q_{11} a_{10}\right)=q_{11}^{\prime} \in Q_{11}$. However, using (4), $\left(b_{10} a_{10}\right) q_{11}=$ $b_{10}\left(a_{10} q_{11}+q_{11} a_{10}\right)-\left(b_{10} q_{11}\right) a_{10}=b_{10}\left(q_{11} a_{10}\right)$, since $R_{10} R_{11}=0$ follows from the table. Comparing the last two equations, $\left(q_{11} a_{10}\right) b_{10}=q_{11}^{\prime}-\left(b_{10} a_{10}\right) q_{11}$. But

$$
\left(b_{10} a_{10}\right) q_{11} \subset\left(R_{10}^{2}\right) Q_{11} \subset\left(R_{11}+R_{01}\right) Q_{11} \subset R_{11} Q_{11}+R_{01} Q_{11} \subset Q_{11}+R_{01} Q_{11} .
$$

Hence $\left(q_{11} a_{10}\right) b_{10} \in Q_{11}+R_{01} Q_{11}$, and thus $\left(Q_{11} R_{10}\right) R_{10} \subset Q_{11}+R_{01} Q_{11}$. Also, $\left(Q_{11} R_{10}\right) R_{01} \subset Q_{11}\left(R_{10} R_{01}+R_{01} R_{10}\right)+\left(Q_{11} R_{01}\right) R_{10} \subset Q_{11} R_{11}+Q_{11} R_{00}+\left(R_{11} R_{01}\right) R_{10}$, as a result of (4). But $Q_{11} R_{11} \subset Q_{11}, Q_{11} R_{00} \subset R_{11} R_{00}=0$, and $\left(R_{11} R_{01}\right) R_{10} \subset Q_{11}$ because of Lemma 8 . Hence $\left(Q_{11} R_{10}\right) R_{01} \subset Q_{11}$.

$$
\begin{aligned}
& \left(Q_{11} R_{10}\right) R_{00} \subset Q_{11}\left(R_{10} R_{00}+R_{00} R_{10}\right) \\
& \quad+\left(Q_{11} R_{00}\right) R_{10} \subset Q_{11} R_{10}+Q_{11} R_{01} \subset Q_{11} R_{10}+R_{11} R_{01},
\end{aligned}
$$

using (4) and the table. Hence $\left(Q_{11} R_{10}\right) R_{00} \subset Q_{11} R_{10}+R_{11} R_{01}$.

$$
\left(R_{01} Q_{11}\right) R_{11} \subset R_{01}\left(Q_{11} R_{11}+R_{11} Q_{11}\right)+\left(R_{01} R_{11}\right) Q_{11} \subset R_{01} Q_{11},
$$

using (4). $\quad\left(R_{01} Q_{11}\right) R_{10} \subset Q . \quad\left(R_{01} Q_{11}\right) R_{00} \subset R_{01} R_{00}$. To handle $\left(R_{01} Q_{11}\right) R_{01}$, we recall from Lemma 4 -(i) that $R_{01} \subset R_{11}+R_{10}^{2}+R_{00} R_{10}$, so that

$$
\left.\left.\left(R_{01} Q_{11}\right) R_{01} \subset\left(R_{11} Q_{11}\right) R_{01}+(] R_{10}^{2}\right] Q_{11}\right) R_{01}+\left(\left[R_{00} R_{10}\right] Q_{11}\right) R_{01} \text {. }
$$

Next we shall work on each of the three terms in the right hand side of (5). Thus $\left(R_{11} Q_{11}\right) R_{01} \subset Q_{11} R_{01} \subset R_{11} R_{01}$, or 
As previously noted, $\left(R_{10}^{2}\right) Q_{11}=R_{10}\left(Q_{11} R_{10}\right) \subset\left(Q_{11} R_{10}\right) R_{10}+Q_{11}$, since $a_{10} b_{10}+$ $b_{10} a_{10} \in Q_{11}$. Thus $\left(\left[R_{10}^{2}\right] Q_{11}\right) R_{01} \subset\left(\left[Q_{11} R_{10}\right] R_{10}\right) R_{01}+Q_{11} R_{01}$. But by use of (4), $\left(\left[Q_{11} R_{10}\right] R_{10}\right) R_{01} \subset\left(Q_{11} R_{10}\right)\left(R_{10} R_{01}+R_{01} R_{10}\right)+\left(\left[Q_{11} R_{10}\right] R_{01}\right) R_{10} \subset\left(Q_{11} R_{10}\right) R_{11}+$ $\left(Q_{11} R_{10}\right) R_{00}+\left(\left[Q_{11} R_{10}\right] R_{01}\right) R_{10}$. We saw previously in the lemma that $\left(Q_{11} R_{10}\right) R_{11}=0,\left(Q_{11} R_{10}\right) R_{00} \subset Q_{11} R_{10}+R_{11} R_{01}$, and $\left(\left[Q_{11} R_{10}\right] R_{01}\right) \subset Q_{11}$. Thus $\left(\left[Q_{11} R_{10}\right] R_{10}\right) R_{01} \subset Q_{11} R_{10}+R_{11} R_{01}$. Putting together the various inclusions we see that

$$
\left(\left[R_{10}^{2}\right] Q_{11}\right) R_{01} \subset Q_{11} R_{10}+R_{11} R_{01} .
$$

Using (4) it follows that

$$
\left(R_{00} R_{10}\right) Q_{11} \subset R_{00}\left(R_{10} Q_{11}+Q_{11} R_{10}\right)+\left(R_{00} Q_{11}\right) R_{10} \subset R_{00}\left(Q_{11} R_{10}\right),
$$

because of the table. But then

$$
\begin{aligned}
& \left(\left[R_{00} R_{10}\right] Q_{11}\right) R_{01} \subset\left(R_{00}\left[Q_{11} R_{10}\right]\right) R_{01} \subset R_{00}\left(\left[Q_{11} R_{10}\right] R_{01}+R_{01}\left[Q_{11} R_{10}\right]\right) \\
& \quad+\left(R_{00} R_{01}\right)\left(Q_{11} R_{10}\right) \subset R_{00}\left(R_{10} R_{01}\right)+R_{00}\left(R_{01} Q_{11} R_{10}\right) \\
& \quad+R_{01} Q_{11} R_{10} \subset R_{00}\left(R_{01} Q_{11} R_{10}\right)+R_{01} Q_{11} R_{10},
\end{aligned}
$$

using (4) and the table. Observe that

$$
\left(R_{00}, R_{01}, R_{10}\right) \subset-\left(R_{00}, R_{10}, R_{01}\right) \subset\left(R_{00} R_{10}\right) R_{01} \subset Q_{00},
$$

using the right alternative identity and Lemma 8. Hence

$$
R_{00}\left(R_{01} Q_{11} R_{10}\right) \subset\left(R_{00} R_{01}\right)\left(Q_{11} R_{10}\right)+Q_{00} \subset R_{01} Q_{11} R_{10}+Q_{00} .
$$

Now piecing together various inclusions we see that

$$
\left(\left[R_{00} R_{10}\right] Q_{11}\right) R_{01} \subset R_{01} Q_{11} R_{10}+Q_{00} \text {. }
$$

By combining (5), (6), (7), and (8) we now see that $\left(R_{01} Q_{11}\right) R_{01} \subset R_{11} R_{01}+$ $Q_{11} R_{10}+R_{01} Q_{11} R_{10}+Q_{00} . \quad\left(R_{01} Q_{11} R_{10}\right) R_{11} \subset R_{00} R_{11}=0 . \quad\left(R_{01} Q_{11} R_{10}\right) R_{10} \subset R_{00} R_{10}$. $\left(R_{01} Q_{11} R_{10}\right) R_{00}=R_{00}\left(R_{01} Q_{11} R_{10}\right)$, as a result of Lemma 6 . However, in the process of establishing (8) we observed that $R_{00}\left(R_{01} Q_{11} R_{10}\right) \subset Q_{00}+R_{01} Q_{11} R_{10}$. Therefore $\left(R_{01} Q_{11} R_{10}\right) R_{00} \subset Q_{00}+R_{01} Q_{11} R_{10}$. Because of (4),

$$
\begin{aligned}
& \left(R_{01} Q_{11} R_{10}\right) R_{01} \subset\left(R_{01} Q_{11}\right)\left(R_{10} R_{01}+R_{01} R_{10}\right) \\
& \quad+\left(\left[R_{01} Q_{11}\right] R_{01}\right) R_{10} \subset\left(R_{01} Q_{11}\right) R_{11}+\left(R_{01} Q_{11}\right) R_{00}+\left(\left[R_{01} Q_{11}\right] R_{01}\right) R_{10} .
\end{aligned}
$$

We established earlier in the proof that $\left(R_{01} Q_{11}\right) R_{11} \subset R_{01} Q_{11},\left(R_{01} Q_{11}\right) R_{00}=0$, $\left(R_{01} Q_{11}\right) R_{01} \subset R_{11} R_{01}+Q_{11} R_{10}+R_{01} Q_{11} R_{10}+Q_{00}$. Hence

$$
\begin{aligned}
& \left(\left[R_{01} Q_{11}\right] R_{01}\right) R_{10} \subset\left(R_{11} R_{01}\right) R_{10}+\left(Q_{11} R_{10}\right) R_{10} \\
& \quad+\left(R_{01} Q_{11} R_{10}\right) R_{10}+Q_{00} R_{10} \subset Q_{11}+R_{01} Q_{11}+R_{00} R_{10},
\end{aligned}
$$

again utilizing inclusions previously established in the proof. There- 
fore $\left(R_{01} Q_{11} R_{10}\right) R_{01} \subset Q_{11}+R_{01} Q_{11}+R_{00} R_{10}$. We have now established half of the necessary inclusions for proving that $Q$ is a right ideal of $R$. The others all follow from reversing subscripts. This completes the proof of the lemma.

Lemma 10. If $Q$ of Lemma 9 is zero, then the table becomes the same as that for alternative rings.

Proof. $Q=0$ implies $R_{11} R_{01}=0=R_{00} R_{10}$, as well as $a_{10}^{2}=0=b_{01}^{2}$. Now define $A=R_{01}+R_{01}^{2}+\left(R_{01}^{2}\right) R_{01}+R_{01} R_{10}$. We now proceed to establish that $A$ is a right ideal of $R . R_{01} R_{11} \subset R_{01} . R_{01} R_{10} \subset A . R_{01} R_{01} \subset A . R_{01} R_{00}=0$, follows from the table. Because of (4), $\left(R_{01}^{2}\right) R_{11} \subset R_{01}\left(R_{01} R_{11}+R_{11} R_{01}\right)+$ $\left(R_{01} R_{11}\right) R_{01} \subset R_{01}^{2}$, while $\left(R_{01}^{2}\right) R_{01} \subset A$. Again using (4),

$\left(R_{01}^{2}\right) R_{10} \subset R_{01}\left(R_{01} R_{10}+R_{10} R_{01}\right)+\left(R_{01} R_{10}\right) R_{01} \subset R_{01} R_{00}+R_{01} R_{11}+R_{00} R_{01} \subset R_{01}$ ，

as a result of the table. Again utilizing (4),

$$
\left(R_{01}^{2}\right) R_{00} \subset R_{01}\left(R_{01} R_{00}+R_{00} R_{01}\right)+\left(R_{01} R_{00}\right) R_{01} \subset R_{01}^{2},
$$

because of the table. Again because of (4) and the table

$$
\begin{aligned}
& \left(\left[R_{01}^{2}\right] R_{01}\right) R_{11} \subset\left(R_{01}^{2}\right)\left(R_{01} R_{11}+R_{11} R_{01}\right) \\
& \quad+\left(\left[R_{01}^{2}\right] R_{11}\right) R_{01} \subset\left(R_{01}^{2}\right) R_{01}+\left(\left[R_{01}^{2}\right] R_{11}\right) R_{01}
\end{aligned}
$$

But we just established that $\left(R_{01}^{2}\right) R_{11} \subset R_{01}^{2}$ so that

$$
\left(\left[R_{01}^{2}\right] R_{11}\right) R_{01} \subset\left(R_{01}^{2}\right) R_{01} \text {. }
$$

Thence $\left(\left[R_{01}^{2}\right] R_{01}\right) R_{11} \subset\left(R_{01}^{2}\right) R_{01}$. Again because of (4),

$$
\begin{aligned}
& \left(\left[R_{01}^{2}\right] R_{01}\right) R_{10} \subset\left(R_{01}^{2}\right)\left(R_{01} R_{10}+R_{10} R_{01}\right) \\
& \quad+\left(\left[R_{01}^{2}\right] R_{10}\right) R_{01} \subset\left(R_{01}^{2}\right) R_{00}+\left(R_{01}^{2}\right) R_{11}+\left(\left[R_{01}^{2}\right] R_{10}\right) R_{01} .
\end{aligned}
$$

But we already know that $\left(R_{01}^{2}\right) R_{00} \subset R_{01}^{2},\left(R_{01}^{2}\right) R_{11} \subset R_{01}^{2}$ and $\left(R_{01}^{2} R_{10}\right) \subset R_{01}$, so that $\left(\left[R_{01}^{2}\right] R_{10}\right) R_{01} \subset R_{01}^{2}$. Hence $\left(\left[R_{01}^{2}\right] R_{01}\right) R_{10} \subset R_{01}^{2}$. Since $R_{01}^{2} \subset R_{00}+R_{10}$ follows from the table and $R_{11} R_{01}=0$,

$$
\begin{aligned}
& \left(\left[R_{01}^{2}\right] R_{01}\right) R_{01} \subset\left(\left[R_{10}+R_{00}\right] R_{01}\right) R_{01} \subset\left(R_{11}+R_{01}\right) R_{01} \subset R_{01}^{2} . \\
& \left(\left[R_{01}^{2}\right] R_{01}\right) R_{00} \subset\left(R_{11}+R_{01}\right) R_{00}=0 . \quad\left(R_{01} R_{10}\right) R_{11} \subset R_{00} R_{11}=0 . \\
& \left(R_{01} R_{10}\right) R_{10} \subset R_{00} R_{10}=0 . \\
& \left(R_{01} R_{10}\right) R_{01} \subset R_{00} R_{01} \subset R_{01} .
\end{aligned}
$$

Because of (4), $\left(R_{01} R_{10}\right) R_{00} \subset R_{01}\left(R_{10} R_{00}+R_{00} R_{10}\right)+\left(R_{01} R_{00}\right) R_{10} \subset R_{01} R_{10}$. Thus we have proved that $A$ is a right ideal of $R$. If $A=0$, then $R_{01}=0$. But then we may verify directly that $B=R_{11}+R_{10}$ is a right ideal, for $R_{11}^{2} \subset R_{11}, R_{11} R_{10} \subset R_{10}, R_{11} R_{00}=0, R_{10} R_{11}=0, R_{10} R_{10} \subset R_{11}, R_{10} R_{00} \subset R_{10}$. As $1-e \notin B$, then $B=0$, and $R_{00}=R$. Since $e \notin R_{00}$, this leads to a 
contradiction. Hence we cannot have $A=0$. But then $A=R$. We recall that $A=R_{01}+R_{01}^{2}+\left(R_{01}^{2}\right) R_{01}+R_{01} R_{10}$, while $R_{01}^{2} \subset R_{10}+R_{00}$,

$$
\left(R_{01}^{2}\right) R_{01} \subset\left(R_{10}+R_{00}\right) R_{01} \subset R_{11}+R_{01}, R_{01} R_{10} \subset R_{00} \text {. }
$$

But because the Peirce decomposition is direct, we must have $R_{10} \subset R_{01}^{2}+$ $R_{00}$. And thus $R_{10}^{2} \subset\left(R_{01}^{2}\right) R_{10}+R_{00} R_{10} \subset\left(R_{01}^{2}\right) R_{10}$. But as a result of (4), $\left(R_{01}^{2}\right) R_{10} \subset R_{01}\left(R_{01} R_{10}+R_{10} R_{01}\right)+\left(R_{01} R_{10}\right) R_{01} \subset R_{01} R_{00}+R_{01} R_{11}+R_{00} R_{01} \subset R_{01}$. We have shown that $R_{10}^{2} \subset R_{01}$. By reversing subscripts we also obtain $R_{01}^{2} \subset R_{10}$. This completes the proof of the lemma.

\section{LEMmA 11. $R_{01} Q_{11} R_{10} \subset Q_{00}$ and $R_{10} Q_{00} R_{01} \subset Q_{11}$.}

Proof. Note that $\left(x_{01} q_{11} y_{10}\right)^{2}=-\left(x_{01} q_{11} y_{10}, x_{01}, q_{11} y_{10}\right)+\left(\left[x_{01} q_{11} y_{10}\right] x_{01}\right)$ $\left(q_{11} y_{10}\right)$. Using (3) with $a=x_{01}, b=q_{11} y_{10}, c=x_{01}$, we see that $\left(\left[x_{01} q_{11} y_{10}\right] x_{01}\right)$ $\left(q_{11} y_{10}\right)=x_{01}\left\{\left(\left[q_{11} y_{10}\right] x_{01}\right)\left(q_{11} y_{10}\right)\right\}$. However, $\left(\left[q_{11} y_{10}\right] x_{01}\right)\left(q_{11} y_{10}\right)=\left(q_{11}, y_{10}, x_{01}\right)$ $\left(q_{11} y_{10}\right)+\left(q_{11}\left[y_{10} x_{01}\right]\right)\left(q_{11} y_{10}\right)$. Since $q_{11}\left[y_{10} x_{01}\right] \in R_{11}$, and $\left(R_{11}, R_{11}, R_{10}\right)=0$, we use Lemma 6 to obtain $\left(q_{11}\left[y_{10} x_{01}\right]\right)\left(q_{11} y_{10}\right)=\left(q_{11}^{2}\left[y_{10} x_{01}\right]\right) y_{10}$. Going back to an earlier equation, thus

$$
\left(\left[q_{11} y_{10}\right] x_{01}\right)\left(q_{11} y_{10}\right)=\left(q_{11}, y_{10}, x_{01}\right)\left(q_{11} y_{10}\right)+\left(q_{11}^{2}\left[y_{10} x_{01}\right]\right) y_{10},
$$

and hence

$$
\begin{aligned}
\left(x_{01} q_{11} y_{10}\right)^{2}= & -\left(x_{01} q_{11} y_{10}, x_{01}, q_{11} y_{10}\right) \\
& +x_{01}\left\{\left(q_{11}, y_{10}, x_{01}\right)\left(q_{11} y_{10}\right)\right\} \\
& +x_{01}\left\{\left(q_{11}^{2}\left[y_{10} x_{01}\right]\right) y_{10}\right\} .
\end{aligned}
$$

We shall now establish that $x_{01} q_{11} y_{10} \in Q_{00}$, by induction on the degree of nilpotency of $q_{11}$. Start off by assuming $q_{11}^{2}=0$. Then (1), with $a=b=q_{11}, c=y_{10}, d=x_{01}$ yields $\left(q_{11}^{2}, y_{10}, x_{01}\right)+\left(q_{11}, q_{11}, y_{10} x_{01}-x_{01} y_{10}\right)=$ $q_{11}\left(q_{11}, y_{10}, x_{01}\right)+\left(q_{11}, y_{10}, x_{01}\right) q_{11}=2 q_{11}\left(q_{11}, y_{10}, x_{01}\right)$ as a result of Lemma 6 . However, the left hand side of the equation is zero, since Lemma 3 implies $\left(R_{11}, R_{11}, R_{11}\right)=0$, and the table implies that $\left(R_{11}, R_{11}, R_{00}\right)=0$. But then $2 q_{11}\left(q_{11}, y_{10}, x_{01}\right)=0$, and so $q_{11}\left(q_{11}, y_{10}, x_{01}\right)=0=\left(q_{11}, y_{10}, x_{01}\right) q_{11}$. Now in the light of this we go back to (9), which may now be rewritten as $\left(x_{01} q_{11} y_{10}\right)^{2}=-\left(x_{01} q_{11} y_{10}, x_{01}, q_{11} y_{10}\right)$. But $-\left(x_{01} q_{11} y_{10}, x_{01}, q_{11} y_{10}\right)=$ $\left(x_{01} q_{11} y_{10}, q_{11} y_{10}, x_{01}\right)=\left(\left[x_{01} q_{11} y_{10}\right]\left[q_{11} y_{10}\right]\right) x_{01}$ as a result of the right alternative identity and the table. Moreover, such an element belongs to $\left(R_{00} R_{10}\right) R_{01} \subset Q_{00}$, as a result of Lemma 8. Thus $\left(x_{01} q_{11} y_{10}\right)^{2} \in Q_{00}$. But then it is obvious that $x_{01} q_{11} y_{10} \in Q_{00}$.

Assume inductively that $x_{01} q_{11} y_{10} \in Q_{00}$ whenever the degree of nilpotency of $q_{11}$ is $k<n$ and let us then consider the case when $q_{11}$ has degree of nilpotency $n$. As before, the proof that

$$
-\left(x_{01} q_{11} y_{10}, x_{01} q_{11}, y_{10}\right) \in Q_{00}
$$


goes over. Now $\left(q_{11}, y_{10}, x_{01}\right) \in R_{11}$ so that $\left(q_{11}, y_{10}, x_{01}\right) q_{11} \in R_{11}$. Lemmas 3 and 6 imply that $R_{11}$ is both associative and commutative so that $\left[\left(q_{11}, y_{10}, x_{01}\right) q_{11}\right]^{2}=\left(q_{11}, y_{10}, x_{01}\right)^{2} q_{11}^{2}$. However, as a result of Lemma 8, we have $\left(q_{11}, y_{10}, x_{01}\right)^{2}=0$, so that $\left(q_{11}, y_{10}, x_{01}\right) q_{11} \in Q_{11}$ and its degree of nilpotency is 2. But then by the previous calculation, or for that matter by the induction hypothesis, it becomes clear that

$$
x_{01}\left\{\left[\left(q_{11}, y_{10}, x_{01}\right) q_{11}\right] y_{10}\right\} \in Q_{00} \text {. }
$$

Since $\left(R_{11}, R_{11}, R_{10}\right)=0$ has already been established,

$$
x_{01}\left\{\left(q_{11}, y_{10}, x_{01}\right)\left(q_{11} y_{10}\right)\right\} \in Q_{00} \text {. }
$$

Finally, $\left[q_{11}^{2}\left(y_{10} x_{01}\right)\right]^{[(n+1) / 2]}=0$, and $[(n+1) / 2]<n$ in our situation, so that we may use the induction hypothesis to obtain that

$$
x_{01}\left\{\left(q_{11}^{2}\left[y_{10} x_{01}\right]\right) y_{10}\right\} \in Q_{00} .
$$

Now going back to (9) we see that $\left(x_{01} q_{11} y_{10}\right)^{2} \in Q_{00}$, since $Q_{00}$ is closed under addition. But then $x_{01} q_{11} y_{10} \in Q_{00}$, as before and the induction is completed. This proves $R_{01} Q_{11} R_{10} \subset Q_{00}$. By reversing subscripts we obtain the second part. This completes the proof of the lemma.

LEMMA $12 . \quad\left(Q_{11} R_{01}\right) R_{01} \subset Q_{11}$ and $\left(Q_{00} R_{10}\right) R_{10} \subset Q_{00}$.

Proof. Let $q_{11} \in Q_{11}$ and $a_{01}, b_{01}, x_{01}, y_{01} \in R_{01}$. Then because of (4), $\left(q_{11} a_{01}\right) b_{01}=q_{11}\left(a_{01} b_{01}+b_{01} a_{01}\right)-\left(q_{11} b_{01}\right) a_{01}$. But $q_{11}\left(a_{01} b_{01}+b_{01} a_{01}\right) \in R_{11} R_{00}=0$. Thus

$$
\left(q_{11} a_{01}\right) b_{01}=-\left(q_{11} b_{01}\right) a_{01} .
$$

Now $\left(\left[q_{11} x_{01}\right] y_{01}\right)^{2}=-\left(\left[q_{11} x_{01}\right] y_{01}, q_{11} x_{01}, y_{01}\right)+\left(\left\{\left[q_{11} x_{01}\right] y_{01}\right\}\left\{q_{11} x_{01}\right\}\right) y_{01}$. But as a result of Lemma $8,-\left(\left[q_{11} x_{01}\right] y_{01}, q_{11} x_{01}, y_{01}\right) \in\left(R_{11}, R_{10}, R_{01}\right) \subset Q_{11}$. Hence let $-\left(\left[q_{11} x_{01}\right] y_{01}, q_{11} x_{01}, y_{01}\right)=q_{11}^{\prime}$. On the other hand (3) implies that $\left(\left\{\left[q_{11} x_{01}\right] y_{01}\right\}\left\{q_{11} x_{01}\right\}\right) y_{01}=\left(q_{11} x_{01}\right)\left(\left[y_{01}\left(q_{11} x_{01}\right)\right] y_{01}\right)$. But then apply (10) with $a_{01}=x_{01}, b_{01}=\left[y_{01}\left(q_{11} x_{01}\right)\right] y_{01}$. Thus

$$
\begin{aligned}
& \left(q_{11} x_{01}\right)\left(\left[y_{01}\left(q_{11} x_{01}\right)\right] y_{01}\right)=-\left(q_{11}\left\{\left[y_{01}\left(q_{11} x_{01}\right)\right] y_{01}\right\}\right) x_{01} \\
= & \left(q_{11}, y_{01}\left(q_{11} x_{01}\right), y_{01}\right) x_{01}=\left[\left(q_{11}, q_{11} x_{01}, y_{01}\right) y_{01}\right] x_{01}
\end{aligned}
$$

using (2). Now $\left(\left[q_{11} x_{01}\right] y_{01}\right)^{2}=q_{11}^{\prime}+\left[\left(q_{11}, q_{11} x_{01}, y_{01}\right) y_{01}\right] x_{01}$. Let

$$
\left(q_{11}, q_{11} x_{01}, y_{01}\right)=t_{11} \text {. }
$$

Then as a result of Lemma 8 , we have $t_{11}^{2}=0$, and

$$
\left(\left[q_{11} x_{01}\right] y_{01}\right)^{2}=q_{11}^{\prime}+\left(t_{11} y_{01}\right) x_{01} \text {. }
$$

In (11), replace $q_{11}$ by $t_{11}$. Then 


$$
\left(\left[t_{11} x_{01}\right] y_{01}\right)^{2}=S_{11}^{\prime}+\left[\left(t_{11}, t_{11} x_{01}, y_{01}\right) y_{01}\right] x_{01} .
$$

In an arbitrary ring one may verify the Teichmüller identity:

$$
(w x, y, z)-(w, x y, z)+(w, x, y z)=w(x, y, z)+(w, x, y) z .
$$

Hence let $w=x=t_{11}, y=x_{01}$, and $z=y_{01}$. Then

$$
\begin{aligned}
& \left(t_{11}^{2}, x_{01}, y_{01}\right)-\left(t_{11}, t_{11} x_{01}, y_{01}\right)+\left(t_{11}, t_{11}, x_{01} y_{01}\right) \\
= & t_{11}\left(t_{11}, x_{01}, y_{01}\right)+\left(t_{11}, t_{11}, x_{01}\right) y_{01} .
\end{aligned}
$$

Since $t_{11}^{2}=0$, the first term of the left hand side vanishes. Since $\left(t_{11}, t_{11}, x_{01} y_{01}\right) \subset\left(R_{11}, R_{11}, R_{00}+R_{10}\right)=0$, the third term of the left hand side also vanishes. From (1) it follows that $\left(t_{11}^{2}, x_{01}, y_{01}\right)+\left(t_{11}, t_{11},\left(x_{01}, y_{01}\right)\right)=$ $t_{11}\left(t_{11}, x_{01}, y_{01}\right)+\left(t_{11}, x_{01}, y_{01}\right) t_{11}$. But $t_{11}^{2}=0$, while

$$
\left(t_{11}, t_{11},\left(x_{01}, y_{01}\right)\right) \in\left(R_{11}, R_{11}, R_{00}+R_{10}\right)=0,
$$

so the left hand side of the last equation is zero. If we let $\left(t_{11} x_{01}\right) y_{01}=$ $a_{11}$, and $t_{11}\left(x_{01} y_{01}\right)=b_{10}$, then $t_{11}\left(a_{11}-b_{10}\right)+\left(a_{11}-b_{10}\right) t_{11}=0$. But $t_{11} b_{10}=$ $t_{11}\left(t_{11}\left[x_{01} y_{01}\right]\right)=-\left(t_{11}, t_{11}, x_{01} y_{01}\right) \in\left(R_{11}, R_{11}, R_{00}+R_{10}\right)=0$, while $b_{10} t_{11}=0$, from the table. Thus $t_{11} a_{11}+a_{11} t_{11}=0$. Then from Lemma 6 we have $2 t_{11} a_{11}=0$, so that $t_{11} a_{11}=0$. But then $t_{11}\left(t_{11}, x_{01}, y_{01}\right)=t_{11}\left(a_{11}-b_{10}\right)=0$. Thus what remains from the Teichmüller identity is $-\left(t_{11}, t_{11} x_{01}, y_{01}\right)=$ $\left(t_{11}, t_{11}, x_{01}\right) y_{01}$. Substituting this into (12) we see that $\left(\left[z_{11} x_{01}\right] y_{01}\right)^{2}=$ $S_{11}^{\prime}-\left\{\left[\left(t_{11}, t_{11}, x_{01}\right) y_{01}\right] y_{01}\right\} x_{01}=S_{11}^{\prime}-\left\{\left(t_{11}, t_{11}, x_{01}\right) y_{01}^{2}\right\} x_{01}$, as a result of the right alternative identity. But $y_{01}^{2} \in Q_{00}$, as a result of the table, while $\left(t_{11}, t_{11}, x_{01}\right)=-\left(t_{11}, x_{01}, t_{11}\right) \in R_{10}$. Thus $-\left\{\left(t_{11}, t_{11}, x_{01}\right) y_{00}^{2}\right\} x_{01} \in R_{10} Q_{00} R_{01}$. But as a result of Lemma $11, R_{10} Q_{00} R_{01} \subset Q_{11}$. Thus $\left(\left[t_{11} x_{01}\right] y_{01}\right)^{2} \in Q_{11}$. But then $\left(t_{11} x_{01}\right) y_{01} \in Q_{11}$. Now we may go back to (11) and obtain $\left(\left[q_{11} x_{01}\right] y_{01}\right)^{2} \in Q_{11}$ and so $\left(q_{11} x_{01}\right) y_{01} \in Q_{11}$. We have shown that

$$
\left(Q_{11} R_{01}\right) R_{01} \subset Q_{11} \text {. }
$$

By reversing subscripts we obtain $\left(Q_{00} R_{10}\right) R_{10} \subset Q_{00}$.

This completes the proof of the lemma.

LEMmA 13. $S=Q_{11}+R_{01} Q_{11}+Q_{11} R_{10}+Q_{11} R_{01}+Q_{00}+R_{10} Q_{00}+Q_{00} R_{01}+$ $Q_{00} R_{10}$, is a right ideal of $R$.

Proof. We observe that $Q$, as defined in Lemma 9, has six of the eight terms appearing in $S$. Indeed we can extract the following inclusions directly from the proof of Lemma 9.

$$
\begin{aligned}
& Q_{11} R_{11} \subset Q_{11}, Q_{11} R_{00}=0,\left(R_{01} Q_{11}\right) R_{11} \subset R_{01} Q_{11}, \\
& \left(R_{01} Q_{11}\right) R_{01} \subset Q_{11} R_{01}+Q_{11} R_{10}+R_{01} Q_{11} R_{10}+Q_{00},\left(R_{01} Q_{11}\right) R_{00}=0, \\
& \left(Q_{11} R_{10}\right) R_{11}=0,\left(Q_{11} R_{10}\right) R_{10} \subset Q_{11}+R_{01} Q_{11}, \\
& \left(Q_{11} R_{10}\right) R_{01} \subset Q_{11},\left(Q_{11} R_{10}\right) R_{00} \subset Q_{11} R_{10}+Q_{11} R_{01} .
\end{aligned}
$$


Also because of Lemma $11, R_{01} Q_{11} R_{10} \subset Q_{00}$, so that in fact

$$
\left(R_{01} Q_{11}\right) R_{01} \subset Q_{11} R_{01}+Q_{11} R_{10}+Q_{00} \subset S \text {. }
$$

Besides, $Q_{11} R_{10} \subset S$, and $Q_{11} R_{01} \subset S$. Thus we have proved that

$$
\left(Q_{11}+R_{01} Q_{11}+Q_{11} R_{10}\right) R \subset S .
$$

Then $\left(Q_{11} R_{01}\right) R_{11} \subset R_{10} R_{11}=0$, because of the table. $\left(Q_{11} R_{01}\right) R_{10} \subset Q_{11}$, as a consequence of Lemma 8. As a result of Lemma $12,\left(Q_{11} R_{01}\right) R_{01} \subset Q_{11}$. Using (4) and the table,

$$
\left(Q_{11} R_{01}\right) R_{00} \subset Q_{11}\left(R_{01} R_{00}+R_{00} R_{01}\right)+\left(Q_{11} R_{00}\right) R_{01} \subset Q_{11} R_{01} \subset S .
$$

This completes half of the required number of inclusions. The remaining ones follow by reversing subscripts. This completes the proof of the lemma.

Corollary. $S=0$.

Proof. Assume $S \neq 0$. Then it follows from the lemma that $S=R$. But then from the directness of the Peirce decomposition we must have $Q_{11}=R_{11}$. Since $e \notin Q_{11}$, while $e \in R_{11}$, we have reached a contradiction. Hence, $S=0$.

LEMMA $14 . \quad Q=0$.

Proof. Suppose $Q \neq 0$. Then as a result of Lemma 9, $Q=R$. Since the corollary to Lemma 13 gives us $S=0$, looking at Lemma 9 we see that $R=R_{11} R_{01}+\left(R_{11} R_{01}\right) R_{01}+R_{00} R_{10}+\left(R_{00} R_{10}\right) R_{10}$. Since the Peirce decomposition is direct, then $R_{11} R_{01}=R_{10}$. But from this it follows that $R_{10} R_{10} \subset\left(R_{11} R_{01}\right) R_{10} \subset Q_{11}$, as a result of Lemma 8. But $Q_{11} \subset S=0$, hence $R_{10} R_{10}=0$. At this point form $U=R_{11}+R_{10}$. Then it follows from the table that $R_{11} R_{11} \subset R_{11}, R_{11} R_{10} \subset R_{10}, R_{11} R_{01} \subset R_{10}$, $R_{11} R_{00}=0, R_{10} R_{11}=0, R_{10} R_{10}=0, R_{10} R_{01} \subset R_{11}, R_{10} R_{00} \subset R_{10}$, so that $U$ must be a right ideal. If $U=R$, then $R_{00}=0$, so since $1-e \in R_{00}$, we would have $e=1$, contrary to assumption. On the other hand if $U=0$, then $e=0$, also a contradiction. The contradiction was brought about by supposing $Q \neq 0$. Hence, $Q=0$. This completes the proof of the lemma.

We are now ready to state and prove our main result.

THEOREM. Let $R$ be a right alternative ring without proper right ideals, of characteristic not two. Suppose that $e, 1 \in R$, where $e$ is an idempotent other than 1 , such that $(e, e, R)=0$. Then $R$ must be alternative, hence a Cayley vector matrix algebra of dimension eight over its center. 
Proof. Combining Lemmas 14 and 10, it follows that the table must be the same as that for an alternative ring and that $R_{00}$ and $R_{11}$ have no nilpotent elements. Then it follows from the main theorem of [4] that $R$ must be alternative. However, the reader can get by with proving only Lemmas 14,15 , and 17 of that paper, since Lemma 16 coincides with our Lemma 7. Once $R$ is alternative, the main result of [2] makes $R$ either associative or a Cayley vector matrix algebra. But $R$ cannot be associative, for having an identity element and no proper right ideals force $R$ to be a division ring, which in turn could not have an idempotent $e \neq 1$. This completes the proof of the theorem.

\section{BIBLIOGRAPHY}

1. A. A. Albert, On right alternative algebras, Ann, of Math. 50 (1949), 318-328.

2. __ On simple alternative rings, Canad. J. Math. 4 (1952), 129-135.

3. The structure of right alternative algebras, Ann. of Math. 59 (1954), 408-417.

4. M. M. Humm On a class of right alternative rings without nilpotent ideals, J. Algebra 5 (1967), 164-174.

5. Erwin Kleinfeld, Right alternative rings, Proc. Amer. Math. Soc. 4 (1953), 939-944.

6. On a class of right alternative rings, Math. Zeit. 87 (1965), 12-16.

7. Carl Maneri, Simple $(-1,1)$ rings with an idempotent, Proc. Amer. Math. Soc. 14 (1963), 110-117.

8. R. L. San Soucie, Right alternative division rings of characteristic 2, Proc. Amer. Math. Soc. 6 (1955), 291-296.

9. L. A. Skornyakov, Right alternative fields, Izvestia Akad. Nauk SSSR. Ser. Mat. 15 (1951), 177-184.

Received November 1, 1968. This work was supported in part by a grant from U.S. Army Research (Durham).

UNIVERSITY OF HAWAII AND

UNIVERSITY OF IOWA 


\section{PACIFIC JOURNAL OF MATHEMATICS}

\section{EDITORS}

H. ROYDEN

Stanford University

Stanford, California

Richard Pierce

University of Washington

Seattle, Washington 98105
J. DUGUNDJI

Department of Mathematics

University of Southern California

Los Angeles, California 90007

BASIL GORDON

University of California

Los Angeles, California 90024

\section{ASSOCIATE EDITORS}
E. F. BECKENBACH
B. H. NEUMANN
F. WOLF
K. YosHIDA

\section{SUPPORTING INSTITUTIONS}

UNIVERSITY OF BRITISH COLUMBIA

CALIFORNIA INSTITUTE OF TECHNOLOGY

UNIVERSITY OF CALIFORNIA

MONTANA STATE UNIVERSITY

UNIVERSITY OF NEVADA

NEW MEXICO STATE UNIVERSITY

OREGON STATE UNIVERSITY

UNIVERSITY OF OREGON

OSAKA UNIVERSITY

UNIVERSITY OF SOUTHERN CALIFORNIA
STANFORD UNIVERSITY

UNIVERSITY OF TOKYO

UNIVERSITY OF UTAH

WASHINGTON STATE UNIVERSITY

UNIVERSITY OF WASHINGTON

*

AMERICAN MATHEMATICAL SOCIETY CHEVRON RESEARCH CORPORATION TRW SYSTEMS

NAVAL WEAPONS CENTER 


\section{Pacific Journal of Mathematics}

\section{Vol. 31, No. $1 \quad$ November, 1969}

James Burton Ax, Injective endomorphisms of varieties and schemes........

Richard Hindman Bouldin, A generalization of the Weinstein-Aronszajn

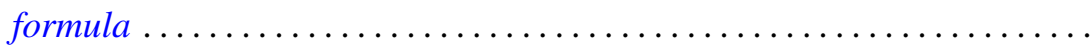

John Martin Chadam, The asymptotic behavior of the Klein-Gordon equation with external potential. II ...............................

Rina Hadass, On the zeros of the solutions of the differential equation

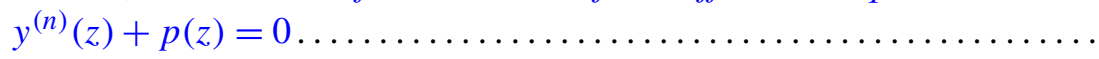

John Sollion Hsia, Integral equivalence of vectors over local modular lattices. II .............................................

Robert Hughes, Boundary behavior of random valued heat polynomial

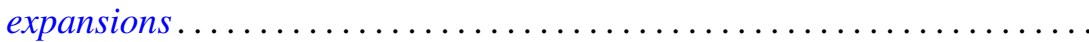

Surender Kumar Jain, Saad H. Mohamed and Surjeet Singh, Rings in which every right ideal is quasi-injective .........................

T. Kawata, On the inversion formula for the characteristic function .........

Erwin Kleinfeld, On right alternative rings without proper right ideals......

Robert Leroy Kruse and David Thomas Price, On the subring structure of

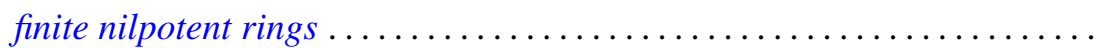

Marvin David Marcus and Stephen J. Pierce, Symmetric positive definite multilinear functionals with a given automorphism. .

William Schumacher Massey, Pontryagin squares in the Thom space of a bundle...

William Schumacher Massey, Proof of a conjecture of Whitney ...

John William Neuberger, Existence of a spectrum for nonlinear transformations

Stephen E. Newman, Measure algebras on idempotent semigroups ...

$\mathrm{K}$. Chandrasekhara Rao, Matrix transformations of some sequence spaces

Robert Bruce Schneider, Some theorems in Fourier analysis on symmetric

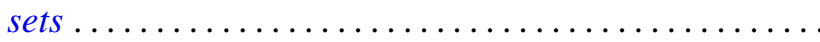

Ulrich F. K. Schoenwaelder, Centralizers of abelian, normal subgroups of hypercyclic groups...

Jerrold Norman Siegel, $G$-spaces, $H$-spaces and $W$-spaces

Robert Irving Soare, Cohesive sets and recursively enumerable Dedekind cuts...

Kwok-Wai Tam, Isometries of certain function spaces .... . .

Awadhesh Kumar Tiwary, Injective hulls of semi-simple modules over regular rings ....

Eldon Jon Vought, Concerning continua not separated by any nonaposyndetic subcontinuum .................... 